\title{
20. Analytic Functions in the Neighbourhood of the Ideal Boundary
}

\author{
By Zenjiro KuRAMochI \\ Mathematical Institute, Hokkaidô University \\ (Comm. by K. Kunugr, M.J.A., Feb. 12, 1957)
}

Let $R$ be a Riemann surface with null-boundary and let $\left\{R_{n}\right\}$ be its exhaustion with compact relative boundary. We proved the following

Theorem 1. ${ }^{1)}$ Let $R^{\prime}$ be a subsurface of $R$ with compact relative boundary. Let $f(z)$ be a bounded analytic function on $R^{\prime}$. Then $f(z)$ has a limit as $z$ tends to an ideal boundary component of $R^{\prime}$.

We extend this theorem to more general class of Riemann surfaces. Let $R$ be a Riemann surface with positive boundary and let $R^{\prime}$ be a subsurface of $R$ with compact relative boundary $\Gamma$. We introduce two classes of Riemann surfaces.

There exists no non-constant one valued bounded (Dirichlet bounded) harmonic function $U(z)$ on $R^{\prime}$ such that $U(z)=0$ on $\Gamma$, the period of the conjugate function of $U(z)$ vanishes along every dividing cut of $R$. We say $R \in O_{A B}^{\prime}$ and $\in O_{A D}^{\prime}$ respectively. $O_{A B}^{\prime}$ and $O_{A D}^{\prime}$ are the extension of the classes of $O_{A B}$ and $O_{A D}$ of the Riemann surface of finite genus. We see easily that the property $\in O_{A B}^{\prime}\left(\in O_{A D}^{\prime}\right)$ is the one depending only on the ideal boundary.

Theorem 2. Suppose a bounded (Dirichlet bounded) analytic function on $R^{\prime} \in \mathrm{O}_{A B}^{\prime}\left(O_{A D}^{\prime}\right)$. Then $f(z)$ has a limit as $z$ tends to a boundary component of $R^{\prime}$.

To prove Theorem 2 we make some preparations.

Let $R$ be a Riemann surface with positive boundary and let $\left\{R_{n}\right\}$ $(n=0,1,2, \cdots)$ be its exhaustion with compact relative boundary $\left\{\partial R_{n}\right\}$. Let $N(z, p): p \in R$ be a positive harmonic function in $R-R_{0}$ such that $N(z, p)=0$ on $\partial R_{0}, N(z, p)$ has a logarithmic singularity at $p$ and $N(z, p)$ has the minimal $*$-Dirichlet integral. ${ }^{2)}$ Let $\left\{p_{i}\right\}$ be a sequence tending to the ideal boundary of $R$ such that $\left\{N\left(z, p_{i}\right)\right\}$ converges uniformly in every compact domain of $R$. We say that $\left\{p_{i}\right\}$ is a fundamental sequence determining an ideal boundary point and we make $\lim _{i=\infty} N\left(z, p_{i}\right)$ correspond to this ideal boundary point. Denote by $B$ the ideal boundary point. The distance between points $p_{1}$ and $p_{2}$ of $R-R_{0}+B$ is defined by

1) Z. Kuramochi: Potential theory and its applications, I, Osaka Math., 3 (1951).

2) Z. Kuramochi: Mass distributions on the ideal boundaries of abstract Riemann surfaces, II, Osaka Math., 8 (1956). 


$$
\delta\left(p_{1}, p_{2}\right)=\sup _{z \in R_{1}-R_{0}}\left|\frac{N\left(z, p_{1}\right)}{1+N\left(z, p_{1}\right)}-\frac{N\left(z, p_{2}\right)}{1+N\left(z, p_{2}\right)}\right| .
$$

Then $R-R_{1}+B$ and $B$ are closed and compact. We defined in the previous paper ${ }^{3)}$ minimal point, singular minimal point. Then we have the following

Lemma 1. Let $p$ be a singular minimal point and let $v(p)$ be a neighbourhood with respect to $\delta$-metric. Then there exists no Dirichlet bounded analytic functions on $v(p)$.

Lemma 2. Suppose $R^{\prime} \in O_{A D}^{\prime}$. Then $R^{\prime}$ has no boundary component of positive capacity.

In fact, assume that $p$ is a boundary component of positive capacity. Then we can construct easily a harmonic function $U(z)$ such that $U(z)=0$ on $\Gamma, D(U(z))<\infty$ and the conjugate of $U(z)$ has no period along every dividing cut.

Lemma 3. Let $U_{n}(z)$ be a harmonic function on $R^{\prime}$ such that $U_{n}(z)=$ Real part of $f(z)$ on $\Gamma$ and $\frac{\partial U_{n}(z)}{\partial n}=0$ on $\partial R_{n}$. Then $U_{n}(z)$ converges to a harmonic function $U^{*}(z)$ in mean and moreover the conjugate of $U(z)$ has no period along every dividing cut, whence $U(z) \equiv \operatorname{Re} f(z) . \quad$ We say such $U(z)$ a $*$-harmonic function. Then we have

Lemma 4. Every $*$-harmonic function satisfies the maximum and minimum principle.

We denote by $\underline{B}$ the all ideal boundary components of $R$. We compactify $R$ by adding $\underline{B}$ to $R$ and introduce usually a topology on $R+\underline{B}$. Then $R+\underline{B}$ and $\underline{B}$ are closed and compact. We call this topology $A$-topology.

Lemma 5. Let $F$ be a closed subset of $R^{\prime}+B$ of capacity zero with respect to A-topology. Then there exists a positive harmonic function $V(z)$ on $R^{\prime}$ such that $V(z)=0$ on $\Gamma, V(z) \rightarrow \infty$ as $z$ tends to $F, V(z)<\infty$ as $z$ tends to a point $\notin F$ and $V_{M}(z)=V(z)$, where $V_{M}(z)$ is a harmonic function such that $V_{M}(z)=0$ on $\Gamma, V_{M}(z)=M$ on $C_{M}=$ $E[z \in R: V(z)=M]$ and has the minimal Dirichlet integral on the domain bounded by $\Gamma$ and $C_{M}$. Hence $\int_{C_{M}} \frac{\partial V(z)}{\partial n} d s \leqq \int_{\Gamma} \frac{\partial V(z)}{\partial n} d s$ for every $0<M<\infty$ and $\int_{C_{M}} \frac{\partial V(z)}{\partial n} d s=\int_{\Gamma^{\prime}} \frac{\partial V(z)}{\partial n} d s$ for every $M \notin E$ such that mes $E=0$.

Remark. In the previous paper ${ }^{5)}$ we proved Lemma 5 under the condition that $F$ is closed in $\delta$-metric and $F \in R+B_{1}$, where $B_{1}$ is the set of minimal points. In this case, the above conditions are not

3)-5) See 2). 
necessary.

Proof of Theorem 2. Suppose $R \in O_{A D}^{\prime}$ and $D(f(z))<\infty$. We denote by $G_{i}$ the domain containing a subset of $\underline{B}$ and bounded by compact or non compact curves $\gamma_{i}$. Denote by $f\left(\gamma_{i}\right)$ the image of $\gamma_{i}$ by $f(z)$. Then by Lemma $4 G_{i} \subset G_{j}$ implies that $f\left(G_{i}\right)$ is contained in $f\left(G_{j}\right)$. Let $p$ be a boundary component of $R^{\prime}$. Apply Lemma 5 to $p$. Then $D(f(z))<\infty$ implies the existence of a sequence of curves $\left\{\gamma_{i}\right\}$ such that $f\left(G_{i}\right)$ is contained in $f\left(\gamma_{i}\right)$ and the length of $f\left(\gamma_{i}\right)$ tends to zero as $i$ tends to $\infty$. Hence $f(z)$ tends to a point $\prod_{i=1}^{\infty} \overline{f\left(G_{i}\right)}{ }^{6}{ }^{6}$

Next suppose $R \in O_{A B}^{\prime}$ and $|f(z)|<M$. We can suppose without loss of generality that $f(z)$ is analytic on $\Gamma$. Consider $U(z)=\operatorname{Re} f(z)$ and $U(z)$ has the minimal Dirichlet integral. Then $U(z) \equiv \operatorname{Re} f(z)$ and $D(U(z))=\int_{\Gamma} U(z) \frac{\partial U(z)}{\partial n} d s$, which implies $D(f(z))<\infty$. On the other hand, we can easily prove $O_{A B}^{\prime} \subset O_{A D}^{\prime}$ by the same method to prove $O_{A B} \subset O_{A D}$ for Riemann surface of genus $O$. Hence $\left|f^{\prime}(z)\right|<M$ and $R^{\prime} \in O_{A B}^{\prime}$ imply $D(f(z))<\infty$ and $R^{\prime} \in O_{A B}^{\prime}$. Thus we have Theorem 2.

6) $\overline{f(G)}$ means the closure of $f(G)$. 